\title{
Association Between the Angiotensin-Converting Enzyme I/D Polymorphism and Risk of Lower-Limb Amputation in Patients with Long-Standing Type 1 Diabetes
}

\author{
Kamel Mohammedi, Yawa Abouleka, Charlyne Carpentier, Louis Potier, Severine Dubois, \\ Ninon Foussard, Vincent Rigalleau, Jean-François Gautier, Pierre Gourdy, Guillaume \\ Charpentier, Ronan Roussel, André Scheen, Bernard Bauduceau, \\ Samy Hadjadj, François Alhenc-Gelas, Michel Marre, Gilberto Velho
}

\section{SUPPLEMENTAL MATERIAL}

Supplemental Figure 1. Study Flow Chart

Supplemental Table 1. Characteristics of participants at baseline by cohort membership

Supplemental Table 2. Characteristics of participants at baseline by ACE I/D genotype

Supplemental Table 3. Incidence of minor and major lower-limb amputations during followup by $A C E \mathrm{I} / \mathrm{D}$ genotype

Supplemental Table 4. Prevalence of lower-limb amputation by $A C E$ I/D genotype in users and non-users of ACE inhibitors at baseline

Supplemental Table 5. Prognostic performance of $A C E$ I/D genotype for the stratification of risk of lower-limb amputation

List of Contributors to SURGENE, GENEDIAB, and GENESIS studies 


\section{Supplemental Figure 1. Study Flow Chart}

SURGENE, GENEDIAB and GENESIS cohorts:

1347 participants with type 1 diabetes

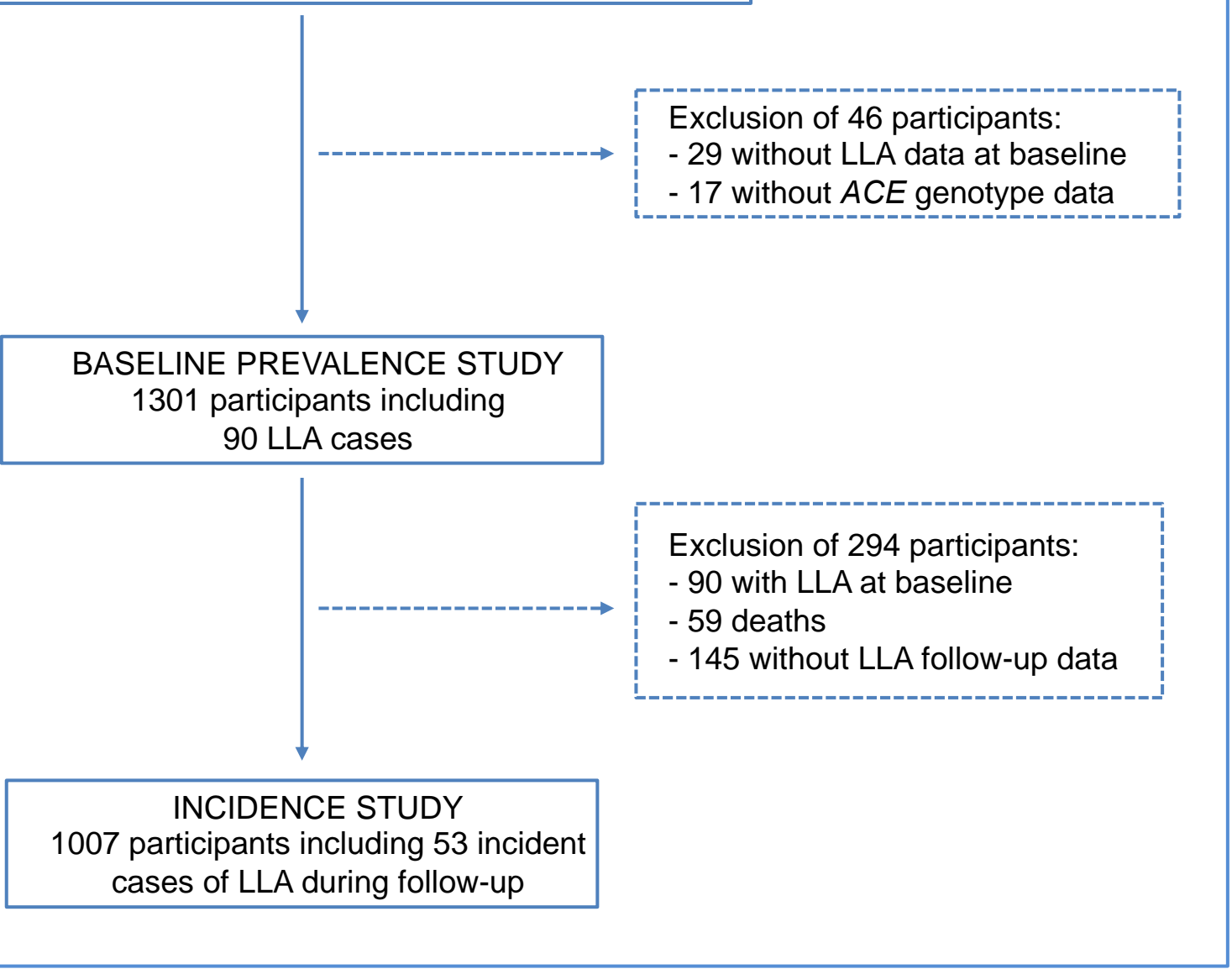


Supplemental Table 1. Characteristics of participants at baseline by cohort membership

\begin{tabular}{|c|c|c|c|c|c|c|}
\hline & \multicolumn{2}{|c|}{ SURGENE } & \multicolumn{2}{|c|}{ GENEDIAB } & \multicolumn{2}{|c|}{ GENESIS } \\
\hline & Data & Missing data (n) & Data & Missing data (n) & Data & Missing data (n) \\
\hline $\mathrm{N}$ & 338 & & 444 & & 519 & \\
\hline Male sex, n (\%) & $190(56)$ & 0 & $249(56)$ & 0 & $269(52)$ & 0 \\
\hline Age (years) & $34 \pm 13$ & 0 & $43 \pm 12$ & 0 & $43 \pm 11$ & 0 \\
\hline Tobacco smoking, n (\%) & NA & NA & & 2 & & 0 \\
\hline Former & - & - & $90(20)$ & & $56(11)$ & \\
\hline Current & - & - & $124(28)$ & & $159(31)$ & \\
\hline Duration of diabetes (years) & $15 \pm 11$ & 0 & $28 \pm 10$ & 1 & $26 \pm 9$ & 1 \\
\hline Body mass index (kg/m2) & $23 \pm 3$ & 0 & $24 \pm 3$ & 3 & $24 \pm 4$ & 5 \\
\hline Systolic blood pressure (mmHg) & $127 \pm 16$ & 3 & $138 \pm 19$ & 0 & $131 \pm 20$ & 15 \\
\hline Diastolic blood pressure $(\mathrm{mmHg})$ & $73 \pm 11$ & 3 & $80 \pm 11$ & 0 & $76 \pm 10$ & 15 \\
\hline $\operatorname{HbA1c}(\%)$ & $9.3 \pm 2.3$ & 2 & $8.6 \pm 1.8$ & 32 & $8.5 \pm 1.3$ & 0 \\
\hline $\mathrm{HbA} 1 \mathrm{c}(\mathrm{mmol} / \mathrm{mol})$ & $79 \pm 25$ & & $71 \pm 20$ & & $70 \pm 14$ & \\
\hline Total cholesterol $(\mathrm{mmol} / \mathrm{l})^{\S}$ & $5.4 \pm 1.3$ & 33 & $5.7 \pm 1.4$ & 5 & NA & \\
\hline eGFR $\left(\mathrm{mL} / \mathrm{min} / 1.73 \mathrm{~m}^{2}\right)$ & $99 \pm 22$ & 4 & $72 \pm 29$ & 0 & $86 \pm 33$ & 5 \\
\hline Urinary albumin concentration $(\mathrm{mg} / \mathrm{l})^{*}$ & $7(3,14)$ & 3 & $42(8,472)$ & 4 & $19(7,169)$ & 11 \\
\hline \multicolumn{7}{|l|}{ History of microvascular disease, $\mathrm{n}(\%)$} \\
\hline Diabetic kidney disease & $58(17)$ & 6 & $266(60)$ & 4 & $241(47)$ & 9 \\
\hline Non-proliferative retinopathy & $94(28)$ & 0 & $85(19)$ & 0 & $323(62)$ & 0 \\
\hline Proliferative retinopathy & $35(10)$ & 0 & $359(81)$ & 0 & $196(38)$ & 0 \\
\hline Peripheral diabetic neuropathy & NA & NA & $297(67)$ & 0 & $191(37)$ & 51 \\
\hline History of macrovascular disease, $\mathrm{n}(\%)$ & & & & & & \\
\hline
\end{tabular}




\begin{tabular}{|c|c|c|c|c|c|c|}
\hline Myocardial infarction & $2(0.6)$ & 1 & $33(7)$ & 1 & $23(4)$ & 0 \\
\hline Stroke & $3(0.8)$ & 0 & $17(4)$ & 4 & $14(3)$ & 4 \\
\hline Peripheral artery disease & NA & NA & $77(17)$ & 0 & $48(9)$ & 59 \\
\hline \multicolumn{7}{|l|}{ Treatments, n $(\%)$} \\
\hline Antihypertensive drugs & $42(12)$ & 0 & $246(57)$ & 12 & $262(50)$ & 0 \\
\hline ACE inhibitors & $29(9)$ & 0 & $148(43)$ & 17 & $191(37)$ & 0 \\
\hline Lipid-lowering drugs & $8(3)$ & 37 & $38(8)$ & 0 & $43(8)$ & 0 \\
\hline$A C E \mathrm{I} / \mathrm{D}$ genotype, $\mathrm{n}(\%)$ & & 0 & & 0 & & 0 \\
\hline II & $56(17)$ & & $82(18)$ & & $83(16)$ & \\
\hline ID & $167(49)$ & & $215(49)$ & & $245(47)$ & \\
\hline DD & $115(34)$ & & $147(33)$ & & $191(37)$ & \\
\hline Plasma ACE $(\mathrm{ng} / \mathrm{ml}) * \dagger$ & $468(350,598)$ & 95 & $437(330,566)$ & 0 & NA & NA \\
\hline
\end{tabular}

Quantitative data presented as mean \pm SD or as *median $\left(25^{\text {th }}-75^{\text {th }}\right.$ percentiles $)$ for those with skewed distribution.

${ }^{\mathfrak{E}}$ Incident lower-limb amputation during follow-up considered in participants without history of LLA at baseline

${ }^{\S}$ Data available in a subset of participants $(\mathrm{N}=744)$

t Data available in a subset of participants $(\mathrm{N}=687)$

NA: Data not available as not collected in SURGENE (smoking, neuropathy and PAD) or in GENESIS (total cholesterol and plasma ACE) 
Supplemental Table 2. Characteristics of participants at baseline by ACE I/D genotype

\begin{tabular}{|c|c|c|c|}
\hline & \multicolumn{2}{|c|}{$A C E$ I/D genotype } & \multirow{2}{*}{$\mathbf{P}$} \\
\hline & XD & II & \\
\hline $\mathrm{N}(\%)$ & $1080(83)$ & $221(17)$ & \\
\hline Cohort membership, n (\%) & & & 0.58 \\
\hline SURGENE & $282(26)$ & $56(25)$ & \\
\hline GENEDIAB & $362(34)$ & $82(37)$ & \\
\hline GENESIS & $436(40)$ & $83(38)$ & \\
\hline Male sex, n (\%) & $587(54)$ & $121(55)$ & 0.94 \\
\hline Age (years) & $40 \pm 13$ & $41 \pm 14$ & 0.26 \\
\hline \multicolumn{4}{|l|}{ Tobacco smoking, n (\%) } \\
\hline Former & $121(11)$ & $25(11)$ & 0.61 \\
\hline Current & $240(22)$ & $43(19)$ & \\
\hline Duration of diabetes (years) & $24 \pm 11$ & $25 \pm 11$ & 0.39 \\
\hline Body mass index (kg/m2) & $24 \pm 3$ & $23 \pm 3$ & 0.13 \\
\hline Systolic blood pressure (mmHg) & $133 \pm 19$ & $132 \pm 18$ & 0.46 \\
\hline Diastolic blood pressure (mmHg) & $77 \pm 11$ & $75 \pm 11$ & 0.20 \\
\hline $\mathrm{HbA} 1 \mathrm{c}(\%)$ & $8.8 \pm 1.8$ & $8.7 \pm 1.7$ & 0.65 \\
\hline $\mathrm{HbA1c}(\mathrm{mmol} / \mathrm{mol})$ & $73 \pm 20$ & $72 \pm 18$ & \\
\hline Total cholesterol $(\mathrm{mmo} / \mathrm{L})^{\S}$ & $5.6 \pm 1.3$ & $5.7 \pm 1.6$ & 0.54 \\
\hline eGFR (mL/min/1.73m²) & $84 \pm 31$ & $86 \pm 30$ & 0.42 \\
\hline Urinary albumin concentration $(\mathrm{mg} / \mathrm{l})^{*}$ & $15(6,156)$ & $12(6,65)$ & 0.13 \\
\hline
\end{tabular}

History of microvascular disease, $\mathrm{n}(\%)$ 


\section{Diabetic kidney disease}

Non-proliferative retinopathy

Proliferative retinopathy

Peripheral diabetic neuropathy

History of macrovascular disease, n (\%)

Myocardial infarction

Stroke

Peripheral artery disease

Treatments, n (\%)

Antihypertensive drugs

ACE inhibitors

Lipid-lowering drugs

$\begin{array}{lcc}480(45) & 85(40) & 0.15 \\ 413(38) & 89(40) & 0.64 \\ 489(45) & 101(46) & \\ 408(38) & 82(37) & 0.60\end{array}$

$\begin{array}{ccc}54(5.0) & 4(1.8) & 0.03 \\ 31(2.9) & 3(1.4) & 0.25 \\ 109(10.1) & 16(7.2) & 0.33\end{array}$

$\begin{array}{lll}470(44) & 80(37) & 0.05 \\ 342(32) & 62(28) & 0.30 \\ 73(7.0) & 16(7.3) & 0.88\end{array}$

$470(359,599) \quad 356(269,445) \quad<0.0001$

Plasma ACE $(\mathrm{mg} / \mathrm{ml}) *+$

Quantitative variables are presented as mean \pm SD or as *median $\left(25^{\text {th }}-75^{\text {th }}\right.$ percentiles $)$ for those with skewed distribution. Comparisons of qualitative and quantitative variables were performed using $\chi^{2}$ and ANOVA tests, respectively. Wilcoxon test was used to compare variables with skewed distribution. $\mathrm{p}<0.05$ was considered as significant.

${ }^{\S}$ Data available for 744 participants.

†Data available for 687 participants. 
Supplemental Table 3. Incidence of minor and major lower-limb amputations during follow-up by $A C E$ I/D genotype

\begin{tabular}{|c|c|c|c|c|c|c|c|}
\hline & \multicolumn{3}{|c|}{ Lower-limb amputation } & \multirow{2}{*}{$\begin{array}{c}\text { Hazard ratio (95\% CI) } \\
\text { for minor LLA vs absent }\end{array}$} & \multirow[t]{2}{*}{$\mathbf{P}$} & \multirow{2}{*}{$\begin{array}{c}\text { Hazard ratio (95\% CI) } \\
\text { for major LLA vs absent }\end{array}$} & \multirow[t]{2}{*}{$\mathrm{P}$} \\
\hline & Absent, $n$ & Minor, n (\%) & Major, n (\%) & & & & \\
\hline \multicolumn{8}{|c|}{$A C E$ genotype } \\
\hline II & 181 & $2(1.1)$ & $3(1.6)$ & 1 & - & 1 & - \\
\hline $\mathrm{XD}$ & 817 & $35(4.0)$ & $23(2.6)$ & $3.63(1.08-22.65)$ & 0.03 & $1.88(0.62-8.28)$ & 0.29 \\
\hline
\end{tabular}

Hazard ratios (with associated 95\% CIs) were computed by Cox model for XD (DD or ID) versus II genotype, adjusted as in model 2: cohort membership, sex, age, history of tobacco smoking, duration of diabetes, HbA1c, systolic and diastolic blood pressure, urinary albumin concentration, estimated glomerular filtration rate, use of ACE inhibitors and use of lipid-lowering drugs. $p<0.05$ was considered as significant. 
Supplemental Table 4. Prevalence of lower-limb amputation by $A C E$ I/D genotype in users and non-users of ACE inhibitors at baseline

\begin{tabular}{|c|c|c|c|c|c|c|c|c|c|}
\hline & \multicolumn{4}{|c|}{ Non users of ACE inhibitors } & \multicolumn{4}{|c|}{ Users of ACE inhibitors } & \multirow{3}{*}{$\begin{array}{c}\text { *P for } \\
\text { interaction }\end{array}$} \\
\hline & \multicolumn{2}{|c|}{ LLA } & \multirow{2}{*}{$\begin{array}{c}\text { Odds ratio } \\
(\mathbf{9 5 \%} \text { CI })\end{array}$} & \multirow{2}{*}{$\mathbf{P}$} & \multicolumn{2}{|c|}{ LLA } & \multirow{2}{*}{$\begin{array}{r}\text { Odds ratio } \\
(95 \% \mathrm{CI})\end{array}$} & \multirow{2}{*}{$\mathbf{P}$} & \\
\hline & No, $n$ & Yes, n (\%) & & & No, $n$ & Yes, n (\%) & & & \\
\hline \multicolumn{10}{|c|}{$A C E$ genotype } \\
\hline II & 152 & $5(3.2)$ & 1 & - & 53 & $9(14.5)$ & 1 & - & \\
\hline XD & 652 & $71(9.8)$ & $5.29(1.80-15.54)$ & 0.002 & 286 & $56(16.4)$ & $1.3(0.60-3.11)$ & 0.46 & 0.08 \\
\hline \multicolumn{10}{|c|}{ Plasma ACE tertiles } \\
\hline $1^{\text {st }}$ tertile & 155 & $24(13.4)$ & 1 & - & 30 & $10(25)$ & 1 & - & \\
\hline $2^{\mathrm{d}}$ tertile & 134 & $20(13.0)$ & $1.14(0.50-2.64)$ & 0.75 & 49 & $19(28)$ & $1.30(0.47-3.57)$ & 0.61 & \\
\hline $3^{\mathrm{d}}$ tertile & 122 & $13(9.6)$ & $0.87(0.33-2.31)$ & 0.78 & 71 & $20(22)$ & $0.86(0.32-2.30)$ & 0.76 & 0.55 \\
\hline
\end{tabular}

Odds ratios computed by logistic regression analyses for the prevalence of lower-limb amputation (LLA) at the end of follow-up by $A C E$ genotype (XD vs II) or tertiles of plasma ACE distribution ( $2^{\mathrm{d}}$ and $3^{\mathrm{d}}$ tertiles vs $1^{\text {st }}$ tertile). Adjustment model: cohort membership, sex, age, history of tobacco smoking, duration of diabetes, $\mathrm{HbA1c}$, systolic and diastolic blood pressure, urinary albumin concentration, estimated glomerular filtration rate, and use of lipid-lowering drugs. *P for interaction between $A C E$ genotype or plasma ACE tertiles and use of ACE inhibitors. p $<0.05$ was considered as significant. 
Supplemental Table 5. Prognostic performance of $A C E$ I/D genotype for the stratification of risk of lower-limb amputation

\begin{tabular}{lccc}
\hline Statistical test & Estimate & $\mathbf{9 5 \%}$ CI & $\mathbf{p}$ \\
\hline Harrell's c-statistic for basic model & 0.791 & $0.752-0.828$ & \\
Change in Harrell's c-statistic for basic model plus ACE I/D genotype & 0.005 & $-0.005-0.014$ & 0.35 \\
IDI & 0.008 & $-0.001-0.024$ & 0.22 \\
Continuous NRI & 0.161 & $0.023-0.303$ & 0.02 \\
\hline
\end{tabular}

Basic model: cohort membership, sex, age, history of tobacco smoking, duration of diabetes, HbA1c, systolic and diastolic blood pressure, urinary albumin concentration, estimated glomerular filtration rate, use of ACE inhibitors and use of lipid-lowering drugs.

Harrell's c-statistic, integrated discrimination improvement (IDI) and net reclassification improvement (NRI) tests were performed for the prevalence of lower-limb amputation at the end of follow-up associated with basic model plus ACE I/D genotype versus basic model alone.

$\mathrm{P}<0.05$ was considered as significant. 


\section{List of Contributors to SURGENE, GENEDIAB, and GENESIS studies}

\section{Clinical investigators at baseline by alphabetical order of enrollment centers}

All cities are in France, except for Liège, in Belgium:

Albert Fournier, Jean-Daniel Lalau (Centre Hospitalier Universitaire d'Amiens); Béatrice Bouhanick, Line Godiveau, Michel Marre, Vincent Rohmer (Centre Hospitalier Universitaire d'Angers); Jean-Raymond Attali, Patrick Miossec (Assistance Publique des Hôpitaux de Paris, Hôpital de Bondy); Henri Gin, Vincent Rigalleau (Centre Hospitalier Universitaire de Bordeaux); Isabelle Cerf, Guillaume Charpentier, Isabelle Petit, Jean-Pierre Riveline (Centre Hospitalier de Corbeil-Essonne); Bertrand Godeau, Zoubida Kahal, Dominique Simon (Assistance Publique des Hôpitaux de Paris, Hôpital de Créteil); Daniel Cordonnier, Serge Halimi (Centre Hospitalier Universitaire de Grenoble); Pierre-Jean Lefebvre, Nicolas Paquot, André Scheen, Laurent Weekers (Centre Hospitalier Universitaire de Liège); Pierre Fontaine, Gaëtan Prevot (Centre Hospitalier Régional Universitaire de Lille); François Berthezene, Fabrice Bonnet, Maurice Laville, Jean-Pierre Fauvel, Charles Thivolet (Assistance Publique des Hôpitaux de Lyon); Bertrand Dussolle, Philippe Vague (Assistance Publique des Hôpitaux de Marseille); Patrick Giraud (Clinique Pont de Chaume, Montauban); Jacques Bringer, Florence Galtier, Michel Rodier (Centres Hospitaliers Universitaires de Montpellier et Nîmes); Pierre Drouin, Laurent Dusselier, Thérèse Crea, Bruno Guerci, Michèle Kessler (Centre Hospitalier Universitaire de Nancy); Lucy Chaillous, Bernard Charbonnel (Centre Hospitalier Universitaire de Nantes); Hamid Boukersi, Françoise Defrance, Etienne Larger, Michel Marre, Ronan Roussel (Assistance Publique des Hôpitaux de Paris, Hôpital Bichat-Claude Bernard); Gérard Slama, Agnès Sola (Assistance Publique des Hôpitaux de Paris, Hôpital de l'Hôtel Dieu); André Grimaldi, Agnès Heurtier, Caroline Sert (Assistance Publique des Hôpitaux de Paris, Hôpital de La Pitié Salpétrière); Jean-Pierre Grunfeld (Assistance Publique des Hôpitaux de Paris, Hôpital Necker); Ahmed Bouallouche, Jean-François Gautier, Pierre-Jean Guillausseau, Hervé Leblanc, Philippe Passa (Assistance Publique des Hôpitaux de Paris, Hôpitaux Saint-Louis et Lariboisière); Samy Hadjadj, Richard Maréchaud, Anne Muller, Florence Torremocha (Centre Hospitalier Universitaire de Poitiers); Jacques Chanard, JeanPaul Melin (Centre Hospitalier Universitaire de Reims); Hubert Allannic, Jean-Yves Poirier (Centre Hospitalier Universitaire de Rennes); Christian Charasse (Centre Hospitalier de SaintBrieuc); Bernard Bauduceau, Lyse Bordier, Hervé Mayaudon (Hôpital d'Instruction des Armées Bégin, Saint-Mandé); Marie-Pierre Arpin-Bott (Centre Hospitalier Universitaire de 
Strasbourg); Hélène Hanaire, Pierre Gourdy, Henri Sackmann, Jean-Pierre Tauber (Centre Hospitalier Universitaire de Toulouse); Odile Verier-Mine (Centre Hospitalier de Valenciennes)

\section{Associated laboratories}

Yves Gallois (Laboratoire de Biochimie Universitaire, Centre Hospitalier Universitaire d'Angers); François Alhenc-Gelas (Centre de Recherche des Cordeliers, INSERM, Université de Paris, Sorbonne Université, Paris)

\section{Statistical Analyses}

Kamel Mohammedi (CHU et université de Bordeaux); Gilberto Velho (Centre de Recherche des Cordeliers, INSERM, Université de Paris, Sorbonne Université, Paris). 